J. Klin. Endokrinol. Stoffw. $2020 \cdot 13: 106-114$ https://doi.org/10.1007/s41969-020-00107-8 Online publiziert: 25 . August 2020

(c) Der/die Autor(en) 2020

Stefan Pilz • Verena Theiler-Schwetz - Oliver Malle · Eva Steinberger · Marlene Pandis · Elisabeth Lerchbaum - Christian Trummer

Klinische Abteilung für Endokrinologie und Diabetologie, Universitätsklinik für Innere Medizin, Medizinische Universität Graz, Graz, Österreich

\title{
Schilddrüse: Fertilität, Schwangerschaft und Laktation
}

bieten eine teils geänderte Evidenzlage ergeben hat, welche zum Zeitpunkt der Veröffentlichung diverser Richtlinien auf diesem Gebiet noch nicht berücksichtigt werden konnte, aber eine praktische Relevanz hat [10].

Bezüglich der zahlreichen physiologischen Veränderungen der Schilddrüse und Schilddrüsenhormone in der Schwangerschaft verweisen wir auf die entsprechende Fachliteratur. Wir möchten jedoch zum praktischen Verständnis anmerken, dass es in der Frühschwangerschaft v. a. durch die Wirkung des humanen Choriongonadotropins (hCG), welches auch den Rezeptor des Thyreoidea-stimulierendem Hormons (TSH) stimuliert, zu einem physiologischen Anstieg der freien Schilddrüsenhormone mit parallelem Abfall des TSH kommt. Daher sind auch in der Schwangerschaft die Normwerte der Schilddrüsenhormone differenziert von den Werten außerhalb der Schwangerschaft zu betrachten [1-5]. Das Thyroxin-bindende Globulin (TBG) steigt ebenfalls, stimuliert u.a. durch Östrogene, in der Schwangerschaft deutlich an, wobei dies weniger relevant für die Routinediagnostik ist, da ohnehin normalerweise die freien (und nicht an TBG gebundenen) Schilddrüsenhormone bestimmt werden.

\section{Fertilität und präkonzeptionelle Phase}

Bei bestehendem Schwangerschaftswunsch wird bei ansonsten gesunden Frauen, welche eine natürliche Konzeption anstreben, aufgrund unzureichender Evidenzlage von diversen Fachgesellschaften keine generelle Empfehlung für oder gegen ein Screening auf Schilddrüsenfunktionsstörungen abgegeben, wobei jedoch in der gängigen Praxis häufig eine Schilddrüsenhormonbestimmung durchgeführt wird [8, 11]. Wir, als Autoren dieses aktuellen Artikels, präferieren trotz der diesbezüglich noch offenen Diskussion im Hinblick auf die Detektion von möglichen bisher unerkannten manifesten Schilddrüsenfunktionsstörungen, die zu ca. $1 \%$ vorliegen, ein generelles (und nicht nur auf bestimmte Risikogruppen beschränktes) Screening aufSchilddrüsenfunktionsstörungen (Bestimmung von TSH plus ggf. freies Thyroxin $\left[\mathrm{fT}_{4}\right]$ ) bei jeder Frau mit Schwangerschaftswunsch und auch bei Erstvorstellung in der Schwangerschaft $[13,14]$. Aufgrund des in der Schwangerschaft erhöhten Jodbedarfs (wegen u. a. vermehrter renaler Clearance, vermehrter Schilddrüsenhormonproduktion und diaplazentarem Jodtransfer) sollte jedoch schon präkonzeptionell auf eine ausreichende Jodversorgung geachtet werden. Dies sollte neben jodhaltigen Nahrungsmitteln auch durch Jodsupplemente mit z.B. $150 \mu$ g Jod pro Tag angestrebt werden, es gibt diesbezüglich aber teils regional unterschiedliche Empfehlungen bzgl. der Joddosis $[8,9,11]$.

\section{Fertilität}

Fertilität und Schilddrüse ist ein häufig diskutiertes Thema, und es zeigte sich u. a. in Beobachtungsstudien ein Zusammenhang zwischen ThyreoperoxidaseAntikörpern (TPO-AK) (Schilddrüsenautoimmunität) und latenter Hypothyreose sowie einem erhöhten Risiko für Infertilität, Aborte (Fehlgeburten) und 
Frühgeburten, wobei die zugrunde liegenden Mechanismen und die Frage der Kausalität derzeit noch unklar sind [2]. Bezüglich eines möglichen kausalen Zusammenhangs zwischen TPO-AK und reduzierter Fertilität gibt es jedenfalls drei gängige Hypothesen: 1) TPO$\mathrm{AK}$ sind ein Epiphänomen einer generellen Autoimmunität, aber nicht der pathogene Faktor; 2) Schilddrüsenautoantikörper sind auch direkt schädlich (pathogen) für die Ovarien (u.a. wurde nachgewiesen, dass Granulosazellen der Ovarien TPO exprimieren); 3) die durch Schilddrüsenautoimmunerkrankungen hervorgerufene Hypothyreose führt zur Infertilität [2]. Durch einen rezenten RCT konnte jedoch geklärt werden, ob eine Therapie mit Levothyroxin (LT4) bei TPO-AK-positiven euthyreoten Frauen mit Infertilität oder stattgehabtem Abortus die Lebendgeburtrate erhöht [15-17]. In dieser Studie (TABLET trial), wurden 952 Frauen randomisiert und erhielten entweder LT4 $50 \mu \mathrm{g}$ tgl. oder Placebo vor und durch die gesamte Schwangerschaft hindurch [15]. Die Lebendgeburtrate war
$37,4 \%$ in der LT4-Gruppe und 37,9\% in der Placebogruppe und zeigte somit ebenso keinen statistisch signifikanten Unterschied wie auch diverse Subgruppenanalysen, wobei hier insbesondere erwähnt sei, dass auch bei den ca. $31 \%$ der Frauen, die ein TSH $>2,5 \mathrm{mIU} / \mathrm{L}$ hatten, kein Unterschied in der Lebendgeburtrate zwischen der LT4-Gruppe versus der Placebogruppe (55 versus 58 Lebendgeburten) bestand [15]. Auch bei Frauen mit assistierter Reproduktion/In-vitro-Fertilisierung (IVF) wurde der Zusammenhang zwischen Schilddrüsenfunktion sowie TPO-AK und Aborten sowie Lebendgeburten intensiv untersucht [18-22]. In diesem Kontext zeigte ein RCT mit 600 euthyreoten, TPO-AK-positiven Frauen, welche sich einer IVF mit Embryotransfer unterzogen, keinen signifikanten Unterschied in der Lebendgeburt- und Abortrate zwischen einer LT4-Gruppe mit 25 bis $50 \mu \mathrm{g}$ tgl. und einer Placebogruppe [18]. Die Empfehlung der ATA-Richtlinie von 2017, wonach man bei euthyreoten TPOAK-positiven Frauen mit vorangegan- genem Abort eine niedrig dosierte LT4Therapie beginnend mit z. B. 25 bis $50 \mu \mathrm{g}$ tgl. erwägen kann, wird durch die oben erwähnten später publizierten Studien somit sehr in Frage gestellt und unsererseits somit auch nicht empfohlen $[8,10]$. Es sollte jedoch bedacht werden, dass die Studien $\mathrm{zu}$ diesem Thema diverse Limitierungen aufwiesen wie z.B. eine fixe eher niedrige LT4-Dosierung, und dass daher noch diverse Wissenslücken bestehen, wodurch sich die diesbezüglichen Empfehlungen eventuell in Zukunft auch nochmals verändern könnten.

\section{Hypothyreose}

Frauen mit einer manifesten Hypothyreose sollte von einer Schwangerschaft abgeraten werden, bis sie eine Euthyreose erreicht haben, da eine manifeste Hypothyreose in der Frühschwangerschaft das Risiko für u. a. Fehlgeburten, Frühgeburten oder neurointellektuelle Störungen des Kindes erhöht [11]. Bei Frauen mit LT4-Therapie und Schwangerschaftswunsch sollte präkonzeptionell ei-

Hier steht eine Anzeige. 
ne TSH-Kontrolle erfolgen, wobei die ATA-Richtlinie 2017 als TSH-Zielbereich einen Wert zwischen dem unteren Grenzwert des Referenzbereichs und 2,5 mIU/L empfiehlt [8]. Hierbei ist zu betonen, dass sich dieser Zielbereich nur auf Frauen bezieht, die schon eine LT4-Therapie mit entsprechender Indikation erhalten, da man bei ihnen davon ausgeht, dass sie im Gegensatz zu gesunden Frauen nur einen eingeschränkten (u.a. hCG-mediierten) Anstieg der freien Schilddrüsenhormone in der Frühschwangerschaft haben. Frauen, die einen Kinderwunsch haben und bereits eine Schilddrüsenhormonsubstitution einnehmen, sollten auch zudem darüber aufgeklärt werden, dass in der Schwangerschaft der Bedarf an Schilddrüsenhormonen um ca. 20-30 \% erhöht ist, d.h., dass sie bei Eintritt der Schwangerschaft in der Regel eine höhere Dosis benötigen $[8,11]$. Empirisch wird hierbei neben einer Bestimmung der Schilddrüsenhormone bei Eintritt der Schwangerschaft häufig empfohlen, noch vor Vorliegen der Schilddrüsenhormonwerte sofort die LT4-Dosis zu erhöhen (Anmerkung: Trijodthyronin $\left[\mathrm{T}_{3}\right]$ wird stärker inaktiviert als $\mathrm{T}_{4}$ bzw. nicht ausreichend in das fötale Zentralnervensystem transferiert, weswegen u. a. in der Schwangerschaft zur Substitution reine $\mathrm{T}_{4}$-Präparate gemäß Leitlinien zu verwenden sind) [8]. Diesbezüglich wird empirisch empfohlen, z. B. 2 mal pro Woche die doppelte LT4-Dosis einzunehmen oder die LT4Dosis um $25 \mu$ g pro Tag zu erhöhen, wenn man bisher LT4-Dosen von bis zu $100 \mu \mathrm{g}$ tgl. eingenommen hat, bzw. die LT4-Dosis um $50 \mu \mathrm{g}$ pro Tag zu erhöhen, wenn man bisher LT4-Dosen von über $100 \mu \mathrm{g}$ tgl. eingenommen hat $[8,11]$.

Eine klinisch häufig auftretende Frage ist, ob Frauen mit bestehendem Kinderwunsch und latenter Hypothyreose mit TSH-Werten $<10 \mathrm{mIU} / \mathrm{L}$ eine LT4-Therapie erhalten sollten (bei höherem TSH wird generell eine Therapie empfohlen). Diesbezüglich ist in Beobachtungsstudien ein Zusammenhang zwischen latenter Hypothyreose und reduzierter Fertilität nicht ganz konsistent, wobei jedoch eine rezente Metaanalyse von RCTs und Kohortenstudien nahelegt, dass eine LT4-Therapie bei latenter Hypothyreose das Abortrisiko reduziert [2, 23, 24]. Die

J. Klin. Endokrinol. Stoffw. 2020 · 13:106-114 https://doi.org/10.1007/s41969-020-00107-8 (c) Der/die Autor(en) 2020

\section{S. Pilz · V. Theiler-Schwetz • O. Malle · E. Steinberger · M. Pandis · E. Lerchbaum · C. Trummer}

\section{Schilddrüse: Fertilität, Schwangerschaft und Laktation}

\section{Zusammenfassung}

Schilddrüsenhormone und Schilddrüsenerkrankungen spielen eine wichtige Rolle bei Fertilität, Schwangerschaft und Laktation. Die diversen Richtlinien und Expertenempfehlungen zur Diagnostik und Therapie von Schilddrüsenerkrankungen bei Kinderwunsch und Schwangerschaft sind teils heterogen und oftmals ohne klare Handlungsanweisung für oder gegen eine bestimmte Maßnahme, was eine gewisse Verunsicherung hervorruft. In diesem Übersichtsartikel möchten wir daher die derzeitige Evidenz auf diesem Gebiet in Bezug auf praktische Handlungsanweisungen darlegen, um dem Leser für die Praxis eine nützliche Entscheidungshilfe an die Hand zu geben. Wir werden besonders auf die aktuelle Evidenzlage bzgl. der Behandlung der latenten Hypothyreose präkonzeptionell, bei Infertilität und in der Schwangerschaft eingehen, sowie auf die

\section{The thyroid: fertility, pregnancy, and lactation}

\section{Abstract}

Thyroid hormones and thyroid diseases play an important role for fertility, pregnancy, and lactation. Various guidelines and expert recommendations regarding the diagnosis and treatment of thyroid disorders in pregnancy and women who wish to conceive are partially inconsistent and often without a clear statement for or against a certain intervention, and are thus often reason for uncertainty. In this brief review we aim to summarize the current evidence on this topic with reference to patient care and we aim to translate the current literature into advice for clinical decisions in daily routine. We focus particularly on the current evidence regarding treatment of subclinical hypothyroidism in the preconception phase and in the context of infertility as well as in pregnancy, and we stress the importance of patient counseling
Wichtigkeit der Beratung und gemeinsamen Therapieentscheidung bei Hyperthyreose und Kinderwunsch bzw. Schwangerschaft. Wir möchten auch besonders betonen, dass diverse wichtige Studien erst nach Publikation der aktuellen Richtlinien auf diesem Gebiet veröffentlicht wurden, was in der Routinebehandlung unserer Patientinnen natürlich berücksichtigt werden sollte. $\mathrm{Da}$ manifeste Schilddrüsenerkrankungen präkonzeptionell und in der Schwangerschaft häufig und in der Regel therapiebedürftig sind, plädieren wir für ein generelles Screening auf Schilddrüsenfunktionsstörungen bei allen Frauen mit Kinderwunsch sowie bei allen mit positivem Schwangerschaftstest.

Schlüsselwörter

Hormone $\cdot$ Richtlinie $\cdot$ Hypothyreose . Hyperthyreose $\cdot$ Thyreostatika and shared decision making regarding hyperthyroidism in the preconception phase as well as in pregnancy. We want to emphasize that several important studies were published after the release of existing guidelines on this topic, which has to be taken into account regarding current decisions for patient care. Considering that overt thyroid diseases are common in women who wish to conceive and who are pregnant, and given that they usually require adequate treatment, we recommend a general screening for thyroid dysfunction in all women who wish to become pregnant and in those with a positive pregnancy test.

\section{Keywords}

Hormones · Guideline · Hypothyroidism .

Hyperthyroidism · Antithyroid drug treatment
ATA-Richtlinie 2017 empfiehlt, dass bei Schwangerschaftswunsch ohne assistierte Reproduktionsmedizin und latenter Hypothyreose mit TSH $<10 \mathrm{mIU} / \mathrm{L}$ eine niedrig dosierte LT4-Therapie mit 25 bis $50 \mu \mathrm{g}$ tgl. erwogen werden sollte, wobei die Autoren dieses Artikels ganz generell eine Frau mit Kinderwunsch und laten- ter Hypothyreose mit TSH $<10 \mathrm{mIU} / \mathrm{L}$ mit z.B. LT4 $50 \mu \mathrm{g}$ tgl. behandeln würden $[2,23,24]$. Bei assistierter Reproduktionsmedizin wird ein Screening mit Schilddrüsenhormonbestimmung allgemein empfohlen, und es sollte bei diesen Frauen im Falle einer latenten Hypothyreose auch eine LT4-Therapie eingeleitet 
werden, da einige kleine RCTs u. a. reduzierte Aborte durch eine Therapie der latenten Hypothyreose in diesem Kontext nachgewiesen haben $[2,23,24]$.

\section{Hyperthyreose}

Bei Frauen mit präkonzeptioneller Hyperthyreose, bei welcher es sich in den meisten Fällen um einen Morbus Basedow handelt, sollte von einer Schwangerschaft abgeraten werden, bis eine Euthyreose erreicht ist, da eine manifeste Hyperthyreose das Risiko u.a. für Fehlgeburten, Frühgeburten und Wachstumsretardierung erhöht [11]. Für das weitere Vorgehen bei Frauen mit Morbus Basedow und laufender thyreostatischer Therapie, welche einen Kinderwunsch haben, gibt es keine klare Empfehlung, es sollte jedoch eine eingehende Aufklärung sowie eine gemeinsame Entscheidung bzgl. der verschiedenen Therapiemöglichkeiten erfolgen $[7,11,12]$. Grundsätzlich gehen die TSH-Rezeptor-Antikörper (TRAK) häufig im Verlauf einer Schwangerschaft, vermutlich im Zuge der Induktion einer Immuntoleranz, zurück, sie können aber postpartal wieder deutlich ansteigen. Zum prinzipiellen Verständnis der Therapie des Morbus Basedow in der Schwangerschaft ist es zudem wichtig zu wissen, dass sowohl die TRAK als auch Thyreostatika die Plazenta passieren und damit auch die kindliche Schilddrüsenhormonproduktion, die etwa $\mathrm{ab}$ der 20. Schwangerschaftswoche (SSW) relevant wird (Beginn der Schilddrüsenhormonsynthese so um die 14 . bis 18. SSW), beeinträchtigen können [7]. Thyreostatika-induzierte Fehlbildungen können auch als mögliche Nebenwirkungen auftreten, wobei diese häufiger bei Thiamazol (Methimazol) (ca. 17 pro 1000 Geburten) als bei Propylthiouracil (Handelsname: Prothiucil ${ }^{(\mathrm{R})}$ ) (ca. 9 pro 1000 Geburten) beobachtet wurden [25]. Daher wird auch Propylthiouracil und nicht Thiamazol als Thyreostatikum im 1. Trimenon bzw. in den ersten 16. SSW empfohlen. Bezüglich der Therapiemöglichkeiten bei Frauen mit Morbus Basedow und Schwangerschaftswunsch hätte die Thyreoidektomie den Vorteil, dass die TRAK danach häufig rasch absinken, wohingegen nach einer Radiojodtherapie die TRAK transient ansteigen mit einem Peak nach ca. 3 Monaten und einer Persistenz über ca. $1 \mathrm{Jahr}$, wodurch sich das Risiko für eine fetale oder neonatale Hyperthyreose erhöhen kann (zudem werden zumindest 6 Monate als Mindestabstand zwischen Radiojodtherapie und Schwangerschaft empfohlen). Falls sich eine Frau für die Fortsetzung der thyreostatischen Therapie trotz bestehendem aktivem Kinderwunsch entscheidet, sollte v. a. bei jungen Frauen, die eine hohe Wahrscheinlichkeit für eine rasche Konzeption haben, schon eine präkonzeptionelle Umstellung von Thiamazol auf Propylthiouracil erwogen werden. Hierbei muss auch bedacht werden, dass außerhalb der Schwangerschaft Thiamazol u.a. wegen des niedrigeren Risikos für ein akutes Leberversagen gegenüber Propylthiouracil bevorzugt wird. Alternativ kann in Erwägung gezogen werden, z. B. bei Frauen mit niedrig dosierter thyreostatischer Therapie (z. B. Thiamazol von maximal $10 \mathrm{mg}$ tgl.), die schon mindestens 6 Monate behandelt wurden und keine große Struma oder hohe TRAK haben, bei Schwangerschaftsnachweis sofort die Thyreostatika abzusetzen und ein engmaschiges Monitoring bzgl. Schilddrüsenfunktion (z.B. alle 1-2 Wochen) in der Frühschwangerschaft anzustreben [7]. Dieses Vorgehen basiert auf der Überlegung, dass das Wiederauftreten einer Hyperthyreose in der Schwangerschaft oft mehrere Wochen braucht, sodass die eventuelle Notwendigkeit für eine thyreostatische Therapie erst wieder nach dem 1 . Trimester besteht und die Therapie somit bis nach der kritischen Phase für mögliche teratogene Nebenwirkungen der Thyreostatika hinausgezögert werden kann.

Frauen, die einen Schwangerschaftswunsch haben und an einer Hyperthyreose aufgrund einer Schilddrüsenautonomie leiden, sollte unbedingt zu einer definitiven Therapie, d.h. entweder Radiojodtherapie oder Operation vor der Schwangerschaft, geraten werden, da sich u. a. die Schilddrüsenautonomie über die Zeit hinweg eher verschlechtert und im Gegensatz zum Morbus Basedow, welcher sich in der Regel in der Schwangerschaft bessert, es keine TRAKgibt, welche bei Notwendigkeit zur thyreostatischen Therapie die Effekte der Thyreostatika auf die kindliche Schilddrüsenfunktion etwas ausgleichen könnten [7].

\section{Schwangerschaft}

\section{Hypothyreose}

In der Schwangerschaft ist jede Hypothyreose mit einem TSH-Wert von über 10,0 mIU/L sowie jede manifeste Hypothyreose mit LT4 zu therapieren (z.B. initial mit einer täglichen Dosis von $2,33 \mu \mathrm{g} / \mathrm{kg}$ oder ca. $150 \mu \mathrm{g}$ ), wobei diese Empfehlung einen generellen weitreichenden Konsensus darstellt, der zwar vor allem auf Beobachtungsstudien basiert, jedoch aufgrund ethischer Bedenken und doch schon überzeugender Evidenzlage vermutlich nicht mehr durch weitere RCTs in Frage gestellt werden wird $[8,9]$.

Bei niedrigeren TSH-Werten sollte gemäß ATA-Richtlinie 2017 jede Frau mit latenter Hypothyreose (d.h. TSHWert über dem trimesterspezifischen oberen Referenzwert) und positiven TPO-AK mit LT4 behandelt werden und eine solche Therapie bei TPO-AKpositiven Frauen mit einem TSH-Wert $>2,5 \mathrm{mIU} / \mathrm{L}$ bis zum oberen TSH-Referenzwert erwogen werden. Bezüglich der TPO-AK wird empfohlen, diese bei jeder Frau in der Schwangerschaft mit einem TSH $>2,5 \mathrm{mIU} / \mathrm{L}$ zu bestimmen, wobei erhöhte TPO-AK bei ca. $2-17 \%$ der schwangeren Frauen detektiert werden und ein erhöhtes Hypothyreoserisiko in der Schwangerschaft anzeigen, weswegen die ATA-Richtlinie 2017 empfiehlt, bei TPO-AK-positiven Frauen (dies gilt auch für Thyreoglobulin-Antikörper [TG-AK]) bis zur Mitte der Schwangerschaft ca. alle 4 Wochen das TSH zu kontrollieren. Für das pathophysiologische Verständnis ist es aber wichtig $\mathrm{zu}$ wissen, dass die TPO-AK zwar die Plazenta passieren, aber die kindliche Schilddrüsenfunktion nicht beeinträchtigen. Die ATA- Richtlinie 2017 empfiehlt für TPO-AK-negative Frauen und bei subklinischer Hypothyreose bis $\mathrm{zu}$ einem TSH-Wert von 10,0 mIU/L, dass man eine Therapie mit LT4 erwägen kann. Diesbezüglich haben 
jedoch neuere RCTs zusätzliche Ergebnisse erbracht, welche zeigten, dass eine LT4-Therapie bei Frauen mit latenter Hypothyreose und Therapiebeginn ab dem 2. Trimenon keine Reduktion von Schwangerschaftskomplikationen oder eine Verbesserung der neurokognitiven Entwicklung der Kinder bewirkt [26-29]. In einem großen MulticenterRCT wurden nach Screening von 97.228 schwangeren Frauen 677 Frauen (SSW: $16+4$ Tage) mit latenter Hypothyreose ( $\mathrm{TSH} \geq 4 \mathrm{mIU} / \mathrm{L}, \mathrm{fT}_{4}$ im Normbereich) $\mathrm{zu}$ entweder LT4 $100 \mu \mathrm{g}$ tgl. oder Placebo randomisiert, wobei monatliche TSH-Kontrollen mit Dosistitration auf einen TSH-Wert von 0,1 bis $2,5 \mathrm{mIU} / \mathrm{L}$ erfolgten [26]. Es zeigten sich bei dieser Studie keinerlei Unterscheide in der neurokognitiven Entwicklung inklusive des Intelligenzquotienten (IQ) der Kinder (primärer Studienendpunkt) im Alter von 5 Jahren, und es gab auch keinerlei signifikante Unterschiede bzgl. diverser Schwangerschaftskomplikationen [26]. In einer Post-hoc-Analyse dieser Studie wurden auch keine Unterscheide zwischen Frauen mit und ohne erhöhten TPO-AK-Wert festgestellt [26]. In der gleichen Studie wurden auch 526 schwangere Frauen (mediane SSW: 18+0) mit einer Hypothyroxinämie, d.h. einer reduzierten $\mathrm{fT}_{4}$-Konzentration bei im Referenzbereich gelegenem TSH-Wert, zu entweder LT4 $50 \mu \mathrm{g}$ tgl. oder Placebo randomisiert, wobei sich ebenfalls keine signifikanten Unterschiede in Bezug auf die neurokognitive Entwicklung bzw. diverse Schwangerschaftskomplikationen zeigte [26]. In der CATS-Studie (Controlled Antenatal Thyroid Screening) wurden 21.846 schwangere Frauen randomisiert in eine Screeninggruppe, in welcher im Falle eines erhöhten TSHund/oder reduzierten $\mathrm{fT}_{4}$-Wertes eine Therapie mit LT4 $150 \mu \mathrm{g}$ tgl. inklusive Dosistitration eingeleitet wurde, und in eine Kontrollgruppe, bei der zwar auch die TSH- und $\mathrm{fT}_{4}$-Werte gemessen wurden, aber keine diesbezügliche Behandlung eingeleitet wurde [29]. In der Screeninggruppe hatten 499 Frauen und in der Kontrollgruppe 551 Frauen ein erhöhtes TSH und/oder reduziertes $\mathrm{fT}_{4}$. Bei 390 Frauen in der Screeninggruppe (mediane SSW: $13+3$ ), welche mit LT4 behandelt wurden, und bei 404 Frauen in der Kontrollgruppe, welche keine diesbezügliche Therapie erhielten, konnte der IQ der Kinder im Alter von 3 Jahren verglichen werden, wobei der mittlere IQ in der Screeninggruppe mit 99,2 nicht signifikant unterschiedlich zum mittleren IQ von 100,0 in der Kontrollgruppe war [29]. Zudem gab es auch bzgl. Schwangerschaftskomplikationen keinen signifikanten Unterschied zwischen diesen beiden Gruppen, und es zeigte eine weitere Untersuchung dieser Studie, dass es auch im Alter von 9,5 Jahren keine signifikanten Gruppenunterschiede bzgl. kognitiver Tests bei den Kindern gab [27]. Eine rezente zusätzliche Auswertung dieser CATS-Studie zeigte jedoch, dass Kinder von mit LT4 behandelten Frauen bei Übertherapie (gekennzeichnet durch erhöhtes $\mathrm{fT}_{4}$ ) signifikant erhöhte Symptome eines Aufmerksamkeitsdefizit-Hyperaktivitäts-Syndroms (ADHS) und weiterer Verhaltensauffälligkeiten zeigten, weswegen die Autoren dieser Arbeit auch betonen, dass auch eine Übertherapie der latenten Hypothyreose in der Schwangerschaft zu Nebenwirkungen führen kann bzw. regelmäßig kontrolliert werden muss [28]. Obwohl auch Subgruppenanalysen der CATSStudie mit Frauen ausschließlich vor der 14. SSW keinen signifikanten Effekt einer Therapie mit LT4 zeigten und man auch schlussfolgerte, dass schwangere Frauen routinemäßig (d.h. in der Praxis) oftmals nicht viel früher in der Schwangerschaft einer Diagnostik und Therapie einer latenten Hypothyreose zugeführt werden, bleibt doch eine gewisse Wissenslücke, ob nicht eine sehr früh in der Schwangerschaft eingeleitete Therapie einer latenten Hypothyreose mit LT4 positive Effekte zeigt. Zu dieser Thematik gibt es aus relativ kleinen und teils nicht Placebo-kontrollierten Studien Hinweise, dass eine sehr früh begonnene Therapie, $d$. h. noch im ersten Trimenon, Schwangerschaftskomplikationen reduzieren könnte [2, 29-32]. Das für die Praxis ableitbare Procedere für die Therapie der latenten Hypothyreose wird auch dadurch erschwert, dass es immer noch keine endgültig klaren und einheitlichen TSH-Grenz- werte in der Schwangerschaft gibt. Die ATA-Richtlinie 2017 empfiehlt diesbezüglich entweder populationsbasierte trimesterspezifische TSH-Grenzwerte oder im ersten Trimester den unteren TSH-Grenzwert um 0,4 mIU/L abzusenken sowie den oberen Grenzwert um $0,5 \mathrm{mIU} / \mathrm{L}$, wodurch sich ein oberer TSH-Grenzwert von ca. 4,0 mIU/L ergeben sollte. Diese Grenzwerte sollen dann graduell im 2. und 3. Trimester an die außerhalb der Schwangerschaft gültigen Grenzwerte angeglichen werden [8]. Die vormals verwendeten, teils noch niedrigeren TSH-Grenzwerte wurden somit durch eine neue Evidenzlage wieder etwas angehoben [1, 8-10].

Um die teilweise Komplexität der Therapie der latenten Hypothyreose in der Schwangerschaft in Bezugnahme auf die aktuelle Evidenzlage für die Praxis zu erleichtern, empfehlen wir folgendes Vorgehen: Alle schwangeren Frauen mit manifester Hypothyreose sowie mit latenter Hypothyreose mit einem TSH-Wert von $\geq 10,0 \mathrm{mIU} / \mathrm{L}$ sind mit LT4 zu therapieren (z. B. initial mit einer täglichen Dosis von $2,33 \mu \mathrm{g} / \mathrm{kg}$ oder ca. $150 \mu \mathrm{g}$ ). Bei einer latenten Hypothyreose im 1. Trimenon mit einem TSH-Wert von $>4,0 \mathrm{mIU} / \mathrm{L}$ bis $<10 \mathrm{mIU} / \mathrm{L}$ sollte unabhängig vom TPO-AK-Status eine Therapie mit z.B. LT4 $50 \mu \mathrm{g}$ tgl. eingeleitet werden (und wir würden auch bei TPO-AK-positiven Schwangeren und einem TSH-Wert von 2,5 bis 4, $0 \mathrm{mIU} / \mathrm{L}$ keine LT4-Therapie einleiten, was auch durch eine rezente Metaanalyse unterstützt wird) [33]. Im 2. und 3. Trimenon kann zwar bei einer latenten Hypothyreose mit einem TSH-Wert von $>4,0 \mathrm{mIU} / \mathrm{L}$ bis $<10 \mathrm{mIU} / \mathrm{L}$ eine Therapie mit z.B. LT4 $50 \mu \mathrm{g}$ tgl. eingeleitet werden, dies kann aber auch gemäß der aktuellen Evidenzlage unterlassen werden (Individualentscheidung, bei der die Autoren bei nur minimalen TSH-Erhöhungen z. B. < 5,0 mIU/L sich eher gegen eine Therapie entscheiden würden).

Bezüglich des Monitorings in der Schwangerschaft empfiehlt die ATARichtlinie von 2017, dass man bei allen Frauen mit latenter Hypothyreose (unabhängig davon, ob behandelt oder nicht), mit manifester Hypothyreose sowie bei euthyreoten schwangeren Frauen, die TPO-AK- und/oder TG-AK-positiv 
sind, ca. alle 4 Wochen eine TSH-Bestimmung bis zur Mitte der Schwangerschaft durchführt und zumindest eine weitere TSH-Bestimmung um ca. die 30. SSW [8]. Der TSH-Zielwert für eine LT4Therapie in der Schwangerschaft sollte natürlich ein im Normbereich liegendes TSH sein, wobei viele Experten und Richtlinien ein TSH $<2,5 \mathrm{mIU} / \mathrm{L}$ in der Schwangerschaft als TSH-Zielbereich anraten.

\section{Hyperthyreose}

Bei einer erstmals in der Schwangerschaft auftretenden Hyperthyreose muss in erster Linie zwischen einer schwangerschaftsinduzierten Hyperthyreose und einem Morbus Basedow unterschieden werden, wobei andere Hyperthyreoseursachen wie z.B. eine Schilddrüsenautonomie in der Schwangerschaft selten sind. Eine schwangerschaftsinduzierte Hyperthyreose wird vor allem durch das hCG verursacht, welches zu einer Stimulation des TSH-Rezeptors führt, und ist häufig auch mit Übelkeit bzw. einer Hyperemesis gravidarum assoziiert. Die schwangerschaftsinduzierte Hyperthyreose tritt im ersten Trimenon auf und normalisiert sich in der Regel spontan (parallel zum hCG-Abfall) ungefähr ab der 15. SSW, wobei die $\mathrm{fT}_{3} / \mathrm{fT}_{4}$-Ratio im Vergleich zum Morbus Basedow niedriger ist und auch die klinische Hyperthyreosesymptomatik (mit Ausnahme der Tachykardie) geringer ist [7]. Im Gegensatz zum Morbus Basedow sind bei der schwangerschaftsinduzierten Hyperthyreose keine TRAK nachweisbar, und das sonografische Muster ist unauffällig. Thyreostatika sollen in der Therapie der schwangerschaftsinduzierten Hyperthyreose nicht (!) verwendet werden (auch meistens nicht bei manifester Hyperthyreose), da es u.a. zu einer spontanen Besserung kommt [8]. Es können jedoch teils supportive Maßnahmen wie Flüssigkeits- und Elektrolytsubstitutionen sowie Beta-Blocker indiziert sein. Der Morbus Basedow zeichnet sich neben dem Vorhandensein von TRAK in der Regel auch durch eine vergrößerte Schilddrüse mit vermehrter Durchblutung und sonografisch echoarmem Muster aus, wobei natürlich auch eine endokrine
Orbitopathie diagnostisch wegweisend sein kann.

Sollte in einer Schwangerschaft eine thyreostatische Therapie bei einem Morbus Basedow notwendig sein, sollten die initialen Dosierungen je nach Höhe der Schilddrüsenhormone bzw. der Symptomatik angepasst werden, wobei meistens Propylthiouracil mit 200 bis $400 \mathrm{mg}$ tgl. oder (seltener, da in den ersten 16 SSW Propylthiouracil bevorzugt verwendet werden sollte; vgl. [34]) Thiamazol mit 10 bis $20 \mathrm{mg}$ tgl. initial verwendet wird (cave: für die Praxis bedenken, dass die Wirksamkeit von Propylthiouracil zu Thiamazol ca. 1:10 bis 20 ist). Sollte eine Frau vor der 16. SSW in der bisherigen Schwangerschaft Thiamazol eingenommen haben und weiterhin eine Thyreostatikatherapie benötigen, wäre prinzipiell in den ersten 16 SSW ein Wechsel auf Propylthiouracil angezeigt, wobei eine rezente Beobachtungsstudie nahelegt, dass in diesem Fall ein Wechsel auf Propylthiouracil die Teratogenität nicht reduziert, weswegen es fraglich ist, ob der Wechsel auf Propylthiouracil unter diesen Umständen sinnvoll wäre [25]. Wichtig dabei ist es, die Thyreostatikadosis so niedrig wie möglich zu halten, wobei als Zielparameter ein $\mathrm{fT}_{4}$-Wert am oder gering über dem oberen Referenzwert angestrebt werden sollte, weil man u. a. auch eine kindliche Hypothyreose vermeiden möchtebzw. Thyreostatikanebenwirkungen auch teils dosisabhängig sind. Schilddrüsenhormonkontrollen sollten dann unter laufender Thyreostatikatherapie initial alle 2-4 Wochen erfolgen bzw. bei Erreichung des $\mathrm{fT}_{4}$ Zielbereichs alle 4-6 Wochen, wobei aufgrund der im Verlauf der Schwangerschaft häufig rückläufigen TRAK die Thyreostatikadosierung regelmäßig reduziert werden bzw. die Thyreostatika häufig (zu ca. 1/3) im 3. Trimester sogar abgesetzt werden können. Ob innerhalb einer Schwangerschaft nach den ersten 16 Wochen von Propylthiouracil wieder auf Thiamazol gewechselt werden soll, ist unklar, und es gibt diesbezüglich keine klare Empfehlung. Jedenfalls wird aber bei allen Patientinnen mit Morbus Basedow (auch wenn schon einer definitiven Therapie zugeführt) ein TRAK-Monitoring in der Schwanger- schaft empfohlen mit Bestimmung der TRAK bei Schwangerschaftsfeststellung und, falls dabei erhöht, nochmals in der 18.-22. SSW und ggf. nochmals in der 30.-34. SSW (falls in der 18.-22. SSW erhöht bzw. eine Thyreostatikatherapie vorliegt). Dies ist empfohlen, da eine TRAK-Erhöhung über das Dreifache des oberen Grenzwertes ein erhöhtes kindliches Hyperthyreoserisiko anzeigt und ein intrauterines und postpartales kindliches Hyperthyreosemonitoring nach sich ziehen sollte.

\section{Postpartale Zeit und Laktation}

\section{Schilddrüsenhormonsubstitution}

Frauen, die im Zuge der Schwangerschaft ihre präkonzeptionell bestehende LT4Dosis aufgrund der Schwangerschaft erhöht haben, sollten unmittelbar nach der Geburt wieder ihre LT4-Therapie auf die präkonzeptionell bestehende Dosis reduzieren mit nachfolgender TSH-Kontrolle in ca. 6 Wochen [8]. Frauen, die erst während der Schwangerschaft erstmals eine LT4-Therapie erhalten haben, sind Kandidatinnen für einen Absetzversuch, vor allem, wenn sie eine geringe Dosis wie z. B. $50 \mu \mathrm{g}$ tgl. einnehmen, wobei dies eine individuelle Entscheidung ist, aber nach Absetzversuch natürlich ca. 6 Wochen später eine TSH-Kontrolle erfolgen sollte [8].

\section{Postpartale Thyreoiditis}

Eine postpartale Thyreoiditis ist eine innerhalb eines Jahres nach der Geburt neu aufgetretene autoimmunologische Schilddrüsendysfunktion, welche nicht einem Morbus Basedow zugeordnet werden kann und bei ca. $5 \%$ aller Frauen auftritt [7]. Frauen mit positiven TPO-AK im ersten Trimenon haben ein erhöhtes Risiko für eine postpartale Thyreoiditis. Typischerweise manifestiert sich diese Erkrankung durch eine ca. 2-6 Monate nach Geburt auftretende transiente Hyperthyreose mit einer Dauer von ca. 1-2 Monaten, welche dann in eine ca. 4- bis 6-monatige hypothyreote Phase übergeht, bevor sich wieder eine Euthyreose einstellt [7]. Es gibt aber auch Verläufe, bei denen nur 


\section{Infobox 1 Ausgewählte Empfehlungen zum Thema Schilddrüse und Schwangerschaft}

- Screening auf Schilddrüsenfunktionsstörungen (Bestimmung von TSH plus ggf. freies Thyroxin [fT $\mathrm{fT}_{4}$ ) bei jeder Frau mit Schwangerschaftswunsch und auch bei Erstvorstellung in der Schwangerschaft

- Präkonzeptionell und in der Schwangerschaft Jodsupplement mit z. B. $150 \mu \mathrm{g}$ Jod pro Tag

- Präkonzeptionell bei im Normbereich liegendem TSH ist keine LT4-Therapieeinleitung erforderlich

- Frau mit Kinderwunsch und latenter Hypothyreose mit TSH $<10 \mathrm{mIU} / \mathrm{L}$ sollte mit z. B. LT4 $50 \mu \mathrm{g} \mathrm{tgl.} \mathrm{behandelt} \mathrm{werden}$

- Frauen mit einer manifesten Hypothyreose sollte von einer Schwangerschaft abgeraten werden, bis sie euthyreot sind

- Bei Frauen mit LT4-Therapie und Schwangerschaftswunsch sollte präkonzeptionell der TSH-Zielbereich zwischen dem unteren Grenzwert des Referenzbereichs und 2,5 mIU/L liegen

- Frauen, die einen Kinderwunsch haben und bereits eine Schilddrüsenhormonsubstitution einnehmen, sollten bei Schwangerschaftseintritt die LT4-Dosis um ca. 20-30\% erhöhen (weitere Therapie dann je nach Laborwerten)

- In der Schwangerschaft ist jede Hypothyreose mit einem TSH-Wert von über 10,0 mIU/L sowie jede manifeste Hypothyreose mit LT4 zu therapieren (z. B. initial mit einer täglichen Dosis von $2,33 \mu \mathrm{g} / \mathrm{kg}$ oder ca. $150 \mu \mathrm{g}$ )

- Bei einer latenten Hypothyreose im 1. Trimenon mit einem TSH-Wert von $>4,0 \mathrm{mIU} / \mathrm{L}$ bis $<10 \mathrm{mIU} / \mathrm{L}$ sollte unabhängig vom TPO-AK-Status eine Therapie mit z. B. LT4 $50 \mu \mathrm{g}$ tgl. eingeleitet werden

- Im 2. und 3. Trimenon kann zwar bei einer latenten Hypothyreose mit einem TSH-Wert von $>4,0 \mathrm{mIU} / \mathrm{L}$ bis $<10 \mathrm{mIU} / \mathrm{L}$ eine Therapie mit z. B. LT4 $50 \mu \mathrm{g} \mathrm{tgl.} \mathrm{eingeleitet} \mathrm{werden,} \mathrm{dies} \mathrm{kann} \mathrm{aber} \mathrm{auch} \mathrm{gemäß} \mathrm{der} \mathrm{aktuellen} \mathrm{Evidenzlage} \mathrm{unterlassen} \mathrm{werden} \mathrm{(Individualentscheidung,} \mathrm{z.} \mathrm{B.} \mathrm{erst} \mathrm{bei} \mathrm{TSH}$ $>5 \mathrm{mlU} / \mathrm{L})$

- Bei einem TSH-Wert von 2,5 bis 4,0 mIU/L in der Schwangerschaft (eher) keine LT4-Therapie

- Bei Frauen mit latenter Hypothyreose (unabhängig davon, ob behandelt oder nicht), mit manifester Hypothyreose sowie bei euthyreoten schwangeren Frauen, die TPO-AK- und/oder TG-AK-positiv sind, sollte ca. alle 4 Wochen eine TSH-Bestimmung bis zur Mitte der Schwangerschaft erfolgen und danach zumindest eine weitere TSH-Bestimmung um die 30. SSW

- Bei Frauen mit präkonzeptioneller Hyperthyreose, bei welcher es sich in den meisten Fällen um einen Morbus Basedow handelt, sollte von einer Schwangerschaft abgeraten werden, bis eine Euthyreose erreicht ist

- Für das weitere Vorgehen bei Frauen mit Morbus Basedow und laufender thyreostatischer Therapie, welche einen Kinderwunsch haben, gibt es keine klare Empfehlung, es sollte jedoch eine eingehende Aufklärung sowie eine gemeinsame Entscheidung bzgl. der verschiedenen Therapiemöglichkeiten erfolgen

- Thyreostatika sollen in der Therapie der schwangerschaftsinduzierten Hyperthyreose nicht verwendet werden (auch meistens nicht bei manifester Hyperthyreose), da es u. a. zur Spontanremission kommt und aufgrund der Thyreostatikanebenwirkungen

- Sollte in einer Schwangerschaft eine thyreostatische Therapie bei einem Morbus Basedow notwendig sein, wird meistens Propylthiouracil mit 200 bis $400 \mathrm{mg}$ tgl. oder (seltener, da in den ersten 16 SSW Propylthiouracil bevorzugt verwendet werden sollte) Thiamazol mit 10 bis $20 \mathrm{mg}$ tgl. initial verwendet

- Sollte eine Frau vor der 16. SSW in der bisherigen Schwangerschaft Thiamazol eingenommen haben und weiterhin eine Thyreostatikatherapie benötigen, wäre prinzipiell in den ersten 16 SSW ein Wechsel auf Propylthiouracil angezeigt, wobei eine Studie nahelegt, dass in diesem Fall ein Wechsel auf Propylthiouracil die Teratogenität nicht reduziert (Individualentscheidung)

- Thyreostatika-Dosis in der Schwangerschaft so niedrig wie möglich halten, wobei als Zielparameter ein $\mathrm{fT}_{4}$-Wert am oder gering über dem oberen Referenzwert angestrebt werden sollte

- Schilddrüsenhormonkontrollen sollten unter laufender Thyreostatikatherapie in der Schwangerschaft initial alle 2-4 Wochen erfolgen bzw. bei Erreichung des $\mathrm{fT}_{4}$-Zielbereiches alle 4-6 Wochen

- TRAK-Monitoring bei Morbus Basedow in der Schwangerschaft: Bestimmung der TRAK bei Schwangerschaftsfeststellung und, falls dabei erhöht, nochmals in der 18.-22. SSW und ggf. nochmals in der 30.-34. SSW (falls in der 18.-22. SSW erhöht bzW. eine Thyreostatikatherapie vorliegt). Eine TRAK-Erhöhung über das Dreifache des oberen Grenzwerts zeigt ein erhöhtes kindliches Hyperthyreoserisiko an und indiziert ein intrauterines und postpartales kindliches Hyperthyreosemonitoring

- Frauen, die im Zuge der Schwangerschaft ihre präkonzeptionell bestehende LT4-Dosis aufgrund der Schwangerschaft erhöht haben, sollten unmittelbar nach der Geburt wieder ihre LT4-Therapie auf die präkonzeptionell bestehende Dosis reduzieren mit nachfolgender TSH-Kontrolle in ca. 6 Wochen

- Eine postpartale Thyreoiditis ist eine innerhalb eines Jahres nach der Geburt neu aufgetretene autoimmunologische Schilddrüsendysfunktion, welche sich typischerweise durch eine ca. 2-6 Monate nach Geburt auftretende transiente Hyperthyreose mit einer Dauer von ca. 1-2 Monaten präsentiert, welche dann in eine ca. 4- bis 6-monatige hypothyreote Phase übergeht, bevor sich wieder eine Euthyreose einstellt; sie ist postpartal ca. 20mal häufiger als ein Morbus Basedow, und es sind Thyreostatika nicht indiziert bzw. ineffektiv; evtl. LT4 erforderlich mit dann Ausschleichversuch nach 12-monatiger Therapie

- Thiamazol bis $20 \mathrm{mg}$ tgl. sowie Propylthiouracil bis $450 \mathrm{mg}$ tgl. sind während der Stillzeit für die Säuglinge sicher, und daher ist keine Kontrolle der kindlichen Schilddrüsenfunktion deswegen notwendig (Thyreostatikasekretion in die Muttermilch ist minimal)

eine Hypo- oder eine Hyperthyreose auftritt. Bei ca. $80 \%$ der Frauen mit postpartaler Thyreoiditis normalisiert sich die Schilddrüsendysfunktion innerhalb eines Jahres, wobei teils auch eine chronische Hypothyreose persistieren kann und die Patientinnen bei einer Folgeschwangerschaft zu ca. $70 \%$ wieder eine postpartale Thyreoiditis ent- wickeln. Differenzialdiagnostisch muss eine postpartale Thyreoiditis von einem Morbus Basedow abgegrenzt werden, wobei bei einer postpartal neu aufgetretenen Hyperthyreose die Inzidenz einer postpartalen Thyreoiditis ca. 20mal gröBer ist als die eines Morbus Basedow. Differenzialdiagnostisch sind TRAK bei einer postpartalen Thyreoiditis im Ge- gensatz zum Morbus Basedow nicht vorhanden bzw. sind Frauen mit einer postpartalen Thyreoiditis meistens TPO-AK-positiv. Das Verhältnis von $\mathrm{fT}_{3}$ $\mathrm{zu} \mathrm{fT} \mathrm{f}_{4}$ ist bei postpartaler Thyreoiditis niedriger als beim Morbus Basedow und die klinische Symptomatik u.a. auch deswegen in der Regel milder. Eine Schilddrüsenszintigrafie ist zur Differen- 
zialdiagnose in den allermeisten Fällen nicht notwendig, aber der Uptake in der Schilddrüsenszintigrafie wäre bei einer postpartalen Thyreoiditis im Gegensatz zum Morbus Basedow in der Regel niedrig bis erniedrigt. In der Therapie der postpartalen Thyreoiditis sind deswegen auch Thyreostatika ineffektiv und nicht indiziert, wobei manchmal bei schwerer Symptomatik der Hyperthyreose eine Behandlung mit Beta-Blockern indiziert sein kann. Eine Hypothyreose im Rahmen einer postpartalen Thyreoiditis wird mit LT4 behandelt, wobei man nach ca. 12-monatiger Therapie einen ausschleichenden Absetzversuch anstreben sollte, um zu evaluieren, ob sich die Schilddrüsenfunktion wieder erholt hat.

\section{Thyreostatika in der Stillzeit}

Es werden zwar sowohl Thiamazol als auch Propylthiouracil in die Muttermilch sezerniert, allerdings sind die Mengen so gering, dass es gemäß diverser Studien bei stillenden Frauen mit Thyreostatikatherapie zu keiner Hypothyreose bei den Säuglingen kam [7]. Man kann daher davon ausgehen, dass Dosierungen von Thiamazol bis $20 \mathrm{mg}$ tgl. sowie von Propylthiouracil bis $450 \mathrm{mg}$ tgl. während der Stillzeit für die Säuglinge sicher sind und daher auch in diesem Kontext keine Kontrolle der kindlichen Schilddrüsenfunktion notwendig ist [7].

Bei jedem Neugeborenen muss jedenfalls das allgemein empfohlene Neugeborenenscreening auf konnatale Hypothyreose in den ersten Tagen nach der Geburt erfolgen [35].

\section{Schlussfolgerungen}

Schilddrüsenerkrankungen sind bei Frauen präkonzeptionell, in der Schwangerschaft und der postpartalen Zeit häufig anzutreffen, wobei man akzeptieren muss, dass es noch immer wissenschaftliche Diskussionen um die jeweiligen Hormongrenzwerte in diesem Kontext gibt und das praktische Vorgehen in der klinischen Routine recht heterogen ist [36-38]. Diverse Beobachtungsstudien haben in der Vergangenheit einen Zusammenhang zwischen einer latenten Hypothyreose bzw. einer TPO-AK-
Positivität und Schwangerschaftskomplikationen gezeigt, und auch die neurokognitive Entwicklung der Kinder in Zusammenhang mit Schilddrüsenerkrankungen wurde intensiv untersucht mit teils unterschiedlichen Ergebnissen [39-45]. In diesem Zusammenhang können wir jedoch nun viele schwangere Frauen insofern beruhigen, dass eben rezente RCTs bei leichten TSHErhöhungen keine signifikanten Effekte einer LT4-Therapie auf Schwangerschaftsverlauf oder neurokognitive Entwicklung der Kinder gezeigt haben $[26-29,33]$. Es hat somit in den letzten Jahren eine Entwicklung von einer vormals sehr aggressiven Therapie der latenten Hypothyreose zu einer aktuell doch deutlich abgeschwächten Therapieempfehlung stattgefunden, wobei es auf diesem Gebiet noch weiterhin einige Wissenslücken gibt. Betonen möchten wir aber andererseits auch die Wichtigkeit der Diagnostik und Therapie manifester Schilddrüsenfunktionsstörungen, welche, mit Ausnahme der schwangerschaftsinduzierten Hyperthyreose, in der Regel unbedingt behandelt werden müssen. Wir plädieren daher auch klar für ein generelles Screening auf Schilddrüsenfunktionsstörungen bei allen Frauen mit Kinderwunsch sowie bei allen mit positivem Schwangerschaftstest.

\section{Korrespondenzadresse}

Assoz. Prof. PD. Dr. Stefan Pilz, PhD

Klinische Abteilung für Endokrinologie und Diabetologie, Universitätsklinik für Innere Medizin, Medizinische Universität Graz Auenbruggerplatz 15, 8036 Graz, Österreich stefan.pilz@chello.at

\section{DDr. Christian Trummer}

Klinische Abteilung für Endokrinologie und Diabetologie, Universitätsklinik für Innere Medizin, Medizinische Universität Graz Auenbruggerplatz 15, 8036 Graz, Österreich christian.trummer@medunigraz.at

Funding. Open access funding provided by Medical University of Graz.

\section{Einhaltung ethischer Richtlinien}

Interessenkonflikt. S. Pilz, V. Theiler-Schwetz, O. Malle, E. Steinberger, M. Pandis, E. Lerchbaum und C. Trummer geben an, dass kein Interessenkonflikt besteht.
Für diesen Beitrag wurden von den Autoren keine Studien an Menschen oder Tieren durchgeführt. Für die aufgeführten Studien gelten die jeweils dort angegebenen ethischen Richtlinien.

Open Access Dieser Artikel wird unter der Creative Commons Namensnennung 4.0 International Lizenz veröffentlicht, welche die Nutzung, Vervielfältigung, Bearbeitung, Verbreitung und Wiedergabe in jeglichem Medium und Format erlaubt, sofern Sie den/die ursprünglichen Autor(en) und die Quelle ordnungsgemäß nennen, einen Link zur Creative Commons Lizenz beifügen und angeben, ob Änderungen vorgenommen wurden.

Die in diesem Artikel enthaltenen Bilder und sonstiges Drittmaterial unterliegen ebenfalls der genannten Creative Commons Lizenz, sofern sich aus der Abbildungslegende nichts anderes ergibt. Sofern das betreffende Material nicht unter der genannten Creative Commons Lizenz steht und die betreffende Handlung nicht nach gesetzlichen Vorschriften erlaubt ist, ist für die oben aufgeführten Weiterverwendungen des Materials die Einwilligung des jeweiligen Rechteinhabers einzuholen.

Weitere Details zur Lizenz entnehmen Sie bitte der Lizenzinformation auf http://creativecommons.org/ licenses/by/4.0/deed.de.

\section{Literatur}

1. Korevaar TIM, Medici M, Visser TJ, Peeters RP (2007) Thyroid disease in pregnancy: new insights in diagnosis and clinical management. Nat Rev Endocrinol 13:610-622

2. Dosiou C (2020) Thyroid and fertility: recent advances. Thyroid 30:479-486

3. Sterrett M (2019) Maternal and fetal thyroid physiology. Clin Obstet Gynecol 62:302-307

4. Springer D, Jiskra J, Limanova Z, Zima T, Potlukova E (2017) Thyroid in pregnancy: from physiology to screening. Crit Rev Clin Lab Sci 54:102-116

5. Iwen KA, Lehnert H (2018) Thyroid and pregnancy. Internist 59:654-660

6. Gronowski AM (2018) Evaluation of thyroid function during pregnancy: Have we taken a wrong turn? Clin Chem 64:439-441

7. Pearce EN (2019) Management of thyrotoxicosis: preconception, pregnancy, and the postpartum period. Endocr Pract 25:62-68

8. Alexander EK, Pearce EN, Brent GA, Brown RS, Chen $\mathrm{H}$, Dosiou $\mathrm{C}$, Grobman WA, Laurberg $\mathrm{P}$, Lazarus JH, Mandel SJ, Peeters RP, Sullivan S (2017) 2017 Guidelines of the American Thyroid Association for the Diagnosis and Management of Thyroid Disease During Pregnancy and the Postpartum. Thyroid 27:315-389

9. Lazarus J, Brown RS, Daumerie C, HubalewskaDydejczyk A, Negro R, Vaidya B (2014) 2014 European Thyroid Association guidelines for the management of subclinical hypothyroidism in pregnancy and in children. EurThyroid J 3:76-94

10. Dong AC, Stephenson MD, Stagnaro-Green AS (2020) The need for dynamic clinical guidelines: a systematic review of new research published after release of the 2017 ATA guidelines on thyroid disease during pregnancy and the postpartum. Front Endocrinol 11:193

11. Okosieme OE, Khan I, Taylor PN (2018) Preconception management of thyroid dysfunction. Clin Endocrinol 89:269-279 
12. Kahaly GJ, Bartalena L, Hegedüs L, Leenhardt L, Poppe K, Pearce SH (2018) 2018 European Thyroid Association guideline for the management of graves' hyperthyroidism. Eur Thyroid J:167-186

13. Stagnaro-Green A, Dong A, Stephenson MD (2019) Universal screening for thyroid disease during pregnancy should be performed. Best Pract Res Clin Endocrinol Metab. https://doi.org/10.1016/j. beem.2019.101320

14. Taylor PN, Zouras S, Min T, Nagarahaj K, Lazarus JH, Okosieme $O$ (2018) Thyroid screening in early pregnancy: pros and cons. Front Endocrinol 9:626

15. Dhillon-Smith RK, Middleton LJ, Sunner KK, Cheed V, Baker K, Farrell-Carver S, Bender-Atik R, Agrawal R, Bhatia K, Edi-Osagie E, Ghobara T, Gupta P, Jurkovic D, Khalaf $Y$, MacLean $M$, McCabe C, Mulbagal K, Nunes N, Overton C, Quenby $S$, Rai R, Raine-Fenning $N$, Robinson $L$, Ross J, Sizer A, Small R, Tan A, Underwood M, Kilby MD, Boelaert K, Daniels J, Thangaratinam S, Chan SY, Coomarasamy A (2019) Levothyroxine in women with thyroid peroxidase antibodies before conception. NEngl J Med 380:1316-1325

16. Chen AX, Leung AM, Korevaar TIM (2019) Thyroid function and conception. N Engl J Med 381:178-181

17. Akhtar MA, Agrawal R, Brown J, Sajjad Y, Craciunas L (2019) Thyroxine replacement for subfertile women with euthyroid autoimmune thyroid disease or subclinical hypothyroidism. Cochrane Database Syst Rev 6:CD11009

18. Wang $\mathrm{H}$, Gao H, Chi H, Zeng L, Xiao W, Wang $Y$, Li R, Liu P, Wang C, Tian Q, Zhou Z, Yang J, Liu Y, Wei R, Mol BWJ, Hong T, Qiao J (2017) Effect of levothyroxine on miscarriage among women with normal thyroid function and thyroid autoimmunity undergoing in vitro fertilization and embryo transfer: a randomized clinical trial. JAMA 318:2190-2198

19. Mintziori G, Goulis DG, Toulis KA, Venetis CA, Kolibianakis EM, Tarlatzis BC (2011) Thyroid function during ovarian stimulation: a systematic review. Fertil Steril 96:780-785

20. Leiva P, Schwarze JE, Vasquez $P$, Ortega C, Villa S, Crosby J, Balmaceda J, Pommer R (2017) There is no association between the presence of antithyroid antibodies and increased reproductive loss in pregnant women after ART: a systematic review and meta-analysis. JBRA Assist Reprod 21:361-365

21. Busnelli A, Paffoni A, Fedele L, Somigliana E (2016) The impact of thyroid autoimmunity on IVF/ICSI outcome: a systematic review and meta-analysis. Hum Reprod Update 22:793-794

22. Simopoulou M, Sfakianoudis K, Maziotis $E$, Grigoriadis S, Giannelou P, Rapani A, Tsioulou P, Pantou A, Kalampokas T, Vlahos N, Pantos K, Koutsilieris M (2019) The impact of autoantibodies on IVF treatment and outcome: a systematic review. Int J Mol Sci 20:E892

23. Maraka S, Singh Ospina NM, Mastorakos G, O'Keeffe DT (2018) Subclinical hypothyroidism in women planning conception and during pregnancy: who should be treated and how? JEndocr Soc 2:533-546

24. Rao M, Zeng Z, Zhou F, Wang H, Liu J, Wang R, Wen Y, Yang Z, Su C, Su Z, Zhao S, Tang L (2019) Effect of levothyroxine supplementation on pregnancy loss and preterm birth in women with subclinical hypothyroidism and thyroid autoimmunity: a systematic review and metaanalysis. Hum Reprod Update 25:344-361

25. Seo GH, Kim TH, Chung JH (2018) Antithyroid drugs and congenital malformations: a nationwide Korean cohort study. Ann Intern Med 168:405-413
26. Casey BM, Thom EA, Peaceman AM, Varner MW, Sorokin Y, HirtzDG, Reddy UM, Wapner RJ, Thorp JM Jr, Saade G, Tita AT, Rouse DJ, Sibai B, lams JD, Mercer BM, Tolosa J, Caritis SN, VanDorsten JP, Eunice Kennedy Shriver National Institute of Child Health and Human Development Maternal-Fetal Medicine Units Network (2017) Treatment of subclinical hypothyroidism or hypothyroxinemia in pregnancy. N Engl J Med 376:815-825

27. Hales C, Taylor PN, Channon S, Paradice R, McEwan K, Zhang L, Gyedu M, Bakhsh A, Okosieme O, Muller I, Draman MS, Gregory JW Dayan C, Lazarus JH, Rees DA, Ludgate M (2018) Controlled antenatal thyroid screening II: effect of treating maternal suboptimal thyroid function on child cognition. J Clin Endocrinol Metab 103:1583-1591

28. Hales C, Taylor PN, Channon S, McEwan K, Thapar A, Langley K, Muller I, Draman MS, Dayan C, Gregory JW, Okosieme O, Lazarus JH, Rees DA, Ludgate M (2020) Controlled antenata thyroid screening II: effect of treating maternal suboptimal thyroid function on child behavior. JClin Endocrinol Metab 105(3):dgz98

29. Lazarus JH, Bestwick JP, Channon S, Paradice $R$ Maina A, Rees R, Chiusano E, John R, Guaraldo V, George LM, Perona M, Dall'Amico D, Parkes AB Joomun M, Wald NJ (2012) Antenatal thyroid screening and childhood cognitive function. NEngl J Med 366:493-501

30. Nazarpour S, Ramezani Tehrani F, Simbar M, Tohidi M, Minooee S, Rahmati M, Azizi F (2018) Effects of levothyroxine on pregnant women with subclinical hypothyroidism, negative for thyroid peroxidase antibodies. J Clin Endocrinol Metab 103:926-935

31. Zhao L, Jiang G, Tian X, Zhang X, Zhu T, Chen B, Wang $Y, M a ~ Q ~(2018)$ Initiation timing effect of levothyroxine treatment on subclinical hypothyroidism in pregnancy. Gynecol Endocrinol 34:845-848

32. Nazarpour $S$, Ramezani Tehrani F, Simbar M Tohidi M, Alavi Majd H, Azizi F (2017) Effects of levothyroxine treatment on pregnancy outcomes in pregnant women with autoimmune thyroid disease. Eur JEndocrinol 176:253-265

33. Sun X, Hou N, Wang H, Ma L, Sun J, Liu Y (2020) A meta-analysis of pregnancy outcomes with levothyroxine treatment in euthyroid women with thyroid autoimmunity. J Clin Endocrinol Metab 105(4):dgz217. https://doi.org/10.1210/clinem/ dgz217

34. https://www.arztnoe.at/fileadmin/Data/ Documents/pdfs/BASG/190122_Thiamazol_ Sandoz.pdf.Zugegriffen: 20.06 .2020

35. Blümel P, Köstl G (2019) Konnatale Hypotthyreose - Diagnose und Behandlungsempfehlung der APEDÖ.J Klin Endokrinol Stoffwechs 12:14-18

36. Maraka S, Mwangi R, McCoy RG, Yao X, Sangaralingham LR, Singh Ospina NM, O'Keeffe DT, De Ycaza AE, Rodriguez-Gutierrez R, Coddington CC 3rd, Stan MN, Brito JP, Montori VM (2017) Thyroid hormone treatment among pregnant women with subclinical hypothyroidism: US national assessment. BMJ 356:i6865

37. Maraka S, Mwangi R, Yao X, Sangaralingham LR, Singh Ospina NM, O'Keeffe DT, RodriguezGutierrez R, Stan MN, Brito JP, Montori VM, McCoy RG (2019) Variation in treatment practices for subclinical hypothyroidism in pregnancy: US national assessment. J Clin Endocrinol Metab. https://doi.org/10.1210/jc.2019-00057

38. Toloza FJK, Singh Ospina NM, Rodriguez-Gutierrez R, O'Keeffe DT, Brito JP, Montori VM, Maraka S
(2019) Practice variation in the care of subclinica hypothyroidism during pregnancy: a national survey of physicians in the United States. J Endocr Soc 3:1892-1906

39. Consortium on Thyroid and Pregnancy-Study Group on Preterm Birth, Korevaar TIM, Derakhshan A, Taylor PN, Meima M, Chen L, Bliddal S, Carty DM, Meems M, Vaidya B, Shields B, Ghafoor F, Popova PV, Mosso L, Oken E, Suvanto E, Hisada A, Yoshinaga J, Brown SJ, Bassols J, Auvinen J, Bramer WM, López-Bermejo A, Dayan C, Boucai L, Vafeiadi M, Grineva EN, Tkachuck AS, Pop VJM, Vrijkotte TG, Guxens M, Chatzi L, Sunyer J, JiménezZabala A, Riaño I, Murcia M, Lu X, Mukhtar S, Delles C, Feldt-Rasmussen U, Nelson SM, Alexander EK, Chaker L, Männistö T, Walsh JP, Pearce EN Steegers EAP, Peeters RP (2019) Association of thyroid function test abnormalities and thyroid autoimmunity with preterm birth: a systematic review and meta-analysis. JAMA 322:632-641

40. Thangaratinam S, Tan A, Knox E, Kilby MD, Franklyn J, Coomarasamy A (2011) Association between thyroid autoantibodies and miscarriage and preterm birth: meta-analysis of evidence. BMJ 342:d2616

41. Drover SSM, Villanger GD, Aase $H$, Skogheim TS, Longnecker MP, Zoeller RT, Reichborn-KjennerudT, Knudsen GP, Zeiner P, Engel SM (2019) Prenatal phthalates, maternal thyroid function, and risk of attention-deficit hyperactivity disorder in the Norwegian mother and child cohort. Epidemiology 30:130-144

42. Levie D, Korevaar TIM, Mulder TA, Bath SC, Dineva M, Lopez-Espinosa MJ, Basterrechea M, Santa-Marina L, Rebagliato M, Sunyer J, Rayman MP, Tiemeier H, Peeters RP, Guxens M (2019) Maternal thyroid function in early pregnancy and child attention-deficit hyperactivity disorder: an individual-participant meta-analysis. Thyroid 29:1316-1326

43. Nelson SM, Haig C, McConnachie A, Sattar N, Ring SM, Smith GD, Lawlor DA, Lindsay RS (2018) Maternal thyroid function and child educational attainment: prospective cohort study. BMJ 360:k452

44. Prezioso G, Giannini C, Chiarelli F (2018) Effect of thyroid hormones on neurons and neurodevelopment. Horm Res Paediatr 90:73-81

45. Jansen TA, Korevaar TIM, Mulder TA, White $T$, Muetzel RL, Peeters RP, Tiemeier H (2019) Maternal thyroid function during pregnancy and child brain morphology: a time window-specific analysis of a prospective cohort. Lancet Diabetes Endocrino 7:629-637

Hinweis des Verlags. Der Verlag bleibt in Hinblick auf geografische Zuordnungen und Gebietsbezeichnungen in veröffentlichten Karten und Institutsadressen neutral. 